Research

\title{
A comparison of the sensitivities of detection of Plasmodium falciparum gametocytes by magnetic fractionation, thick blood film microscopy, and RT-PCR Stephan Karl ${ }^{1}$, Timothy ME Davis ${ }^{2}$ and Tim G St-Pierre*1
}

\author{
Address: ${ }^{1}$ School of Physics, M013, The University of Western Australia, 35 Stirling Highway, Crawley WA 6009, Australia and ${ }^{2}$ School of Medicine \\ and Pharmacology, The University of Western Australia, Fremantle Hospital, Alma Street, Fremantle, WA, Australia \\ Email: Stephan Karl - karls01@student.uwa.edu.au; Timothy ME Davis - tdavis@cyllene.uwa.edu.au; Tim G St- \\ Pierre* - stpierre@physics.uwa.edu.au \\ * Corresponding author
}

Published: II May 2009

Malaria Journal 2009, 8:98 doi:10.1 186/1475-2875-8-98

This article is available from: http://www.malariajournal.com/content/8///98

(C) 2009 Karl et al; licensee BioMed Central Ltd.

This is an Open Access article distributed under the terms of the Creative Commons Attribution License (http://creativecommons.org/licenses/by/2.0), which permits unrestricted use, distribution, and reproduction in any medium, provided the original work is properly cited.
Received: 23 November 2008

Accepted: II May 2009

\begin{abstract}
Background: The magnetic properties of Plasmodium-infected erythrocytes have been exploited for different clinical and research purposes. A recent study in a rural clinical setting in Papua New Guinea has demonstrated that Plasmodium falciparum gametocyte detection is facilitated by magnetic deposition microscopy but no study has yet determined the relative sensitivity and limit of detection of a magnetic fractionation technique. The present study compares the detection limit and sensitivity of a technique based on the use of commercially available magnetic fractionation columns with those for thick blood film microscopy and reverse transcriptase polymerase chain reaction (RT-PCR) methods.
\end{abstract}

Methods: Gametocyte detection in six series of dilutions of cultured $P$. falciparum parasites with known gametocytaemia was conducted using magnetic fractionation, thick blood film, and RT-PCR techniques.

Results: The preparations obtained by the magnetic fractionation method were of thin film quality allowing easy gametocyte identification by light microscopy. Magnetic fractionation had a higher sensitivity and approximately two orders of magnitude better limit of detection than thick blood film microscopy. Gametocytes were also more readily detectable on the magnetically fractionated preparations. Magnetic fractionation had a similar limit of detection to that of RT-PCR.

Conclusion: Magnetic fractionation is a highly sensitive and convenient method for gametocyte detection in comparison with the standard thick blood film and RT-PCR methods, and could readily be adapted to field application.

\section{Background}

Based on the estimated average blood meal of Anopheles gambiae of $1.6 \mu \mathrm{L}$, and the observation that male gametocytes are less numerous than female gametocytes, the lower limit of male gametocyte density in human blood that has epidemiological implications for transmission is estimated to be around $2.5 \mu \mathrm{L}^{-1}[1]$. It has also been reported that gametocyte densities of $1-10 \mu \mathrm{L}^{-1}$ can result in mosquito infection [2]. Detection and gender differentiation of gametocytes on standard thick blood films 
(TBF) at these densities is a challenge even for the experienced microscopist. For confident detection of low-level gametocytaemia molecular methods based on PCR need to be used [3].

Magnetic fractionation (MF) techniques can be used to enrich the concentration of erythrocytic stages of malaria parasites that contain haemozoin, the paramagnetic product of the haem detoxification pathway [4-6]. Commercially available MF columns have been used for various purposes in malaria studies ranging from simple reports confirming the feasibility of magnetically fractionating infected cells, to practical applications such as synchronization of cultures and sample preparation for immunological studies [7-9]. It has been demonstrated that the four main species of Plasmodium that infect humans can be concentrated by magnetic deposition microscopy (MDM), which utilizes a custom-made MF system that is being developed as a re-usable low cost alternative to the single-use MF columns [10]. It was shown recently that Plasmodium falciparum gametocyte detection can also be facilitated by MDM in a rural clinical setting in Papua New Guinea [11].

In the present study, the sensitivity and limit of detection of gametocytes by MF employing commercially available MF columns was compared with those of TBF and reverse transcriptase polymerase chain reaction (RT-PCR) methods. Each method was tested on gametocyte-containing parasite cultures serially diluted with whole blood. The preparations obtained with MF columns in this study are of thin film quality and allow easy identification and differentiation of gametocytes.

\section{Methods}

The laboratory-adapted gametocyte producing $P$. falciparum strains 3D7, Dd2 and W2mef were cultured in RPMI 1640 HEPES (Sigma Aldrich) supplemented with 5 $\mathrm{g} / \mathrm{L}$ albumax II (Invitrogen), $92.6 \mathrm{mg} / \mathrm{L}$ L-glutamine (Sigma Aldrich), $500 \mu \mathrm{g} / \mathrm{L}$ gentamicin (Sigma Aldrich), 50 $\mathrm{mg} / \mathrm{L}$ hypoxanthine (Sigma Aldrich). Culture medium was changed once daily. Cultures were maintained at 4$5 \%$ haematocrit and diluted with red blood cells when parasitaemias exceeded 5\%. Cultures were incubated in an airtight cabinet (Nalgene, Model 53170120 ) at $37^{\circ} \mathrm{C}$ in an atmosphere with approximately $5 \%$ oxygen concentration. The low oxygen atmosphere was generated by gassing the cabinet with a gas mixture $\left(1 \% \mathrm{O}_{2} ; 95 \% \mathrm{~N}_{2} ; 4 \%\right.$ $\mathrm{CO}_{2}$ ) at 10-15 $\mathrm{kPa}$ for 60 seconds each time it had been opened. To induce gametocyte development, the parasite culture was allowed to grow to maximum parasitaemia with gametocytes appearing approximately 10 days later.

For the present experiments, 10 -fold dilution series of the parasite culture were prepared in freshly drawn whole blood. The starting gametocytaemia was determined by counting the number of red blood cells per 100 gametocytes on a thin film prepared from the initial parasite culture. Six experiments were conducted comparing the sensitivities of MF, and RT-PCR (4 experiments using 3D7, 1 experiment using W2mef and 1 experiment using Dd2 strain). The total blood volume prepared for each dilution was $1610 \mu \mathrm{L}$ of which $100 \mu \mathrm{L}$ were used for $\mathrm{MF}$, $10 \mu \mathrm{L}$ to prepare $\mathrm{TBF}$, and $1500 \mu \mathrm{L}$ for RNA extraction and RT-PCR.

\section{Magnetic fractionations}

Magnetic fractionations were conducted using a MidiMACS magnet, a MACS-multistand and LS columns (Miltenyi Biotech). The columns were placed into the magnet unit and primed with $0.7 \mathrm{~mL}$ sterile filtered magnetic fractionation buffer (MFB, PBS pH 7.4, containing $0.5 \mathrm{~g} / \mathrm{L}$ bovine serum albumin and $0.0037 \mathrm{~g} / \mathrm{L}$ EDTA) equilibrated to room temperature. $100 \mu \mathrm{L}$ of blood from each dilution were suspended in $5 \mathrm{~mL}$ MFB in $15-\mathrm{mL}$ centrifuge tubes (BD Biosciences) and incubated at room temperature for at least $10 \mathrm{~min}$ before the start of the fractionation. The flow rate through the column was regulated to $0.25 \mathrm{~mL} / \mathrm{min}$ by attachment of a sterile syringe needle (BD Biosciences) with an inner diameter of 0.42 $\mathrm{mm}(26 \mathrm{G})$ to the end of the column. After the cell suspension had passed through, the columns were washed with $2 \times 1 \mathrm{~mL}$ MFB and removed from the magnet. The sterile needle was disconnected and the cells captured in the column were eluted into $15-\mathrm{mL}$ centrifuge tubes (BD Biosciences) by washing with $1 \times 5 \mathrm{~mL}$ MFB. These fractions were centrifuged at $400 \mathrm{~g}$ for $10 \mathrm{~min}$ (Sigma Laboratory Centrifuge, Model 4K15). The resulting cell pellet was resuspended in approximately $3 \mu \mathrm{L}$ of PBS (pH 7.4) and spread on a microscope slide to form a circle of $0.5-1 \mathrm{~cm}$ in diameter. The slides were dried in an incubator (Sanyo, Model: MCO -15A) at $37^{\circ} \mathrm{C}$ for at least $30 \mathrm{~min}$, fixed with methanol and stained with 5\% Giemsa solution (SigmaAldrich) in PBS (pH 7.4) for 10 min. Leukocyte density was determined by counting the number of leukocytes in 100 high power fields across the MF preparation. Gametocyte density was determined by counting the number of gametocytes per leukocyte in 100 high power fields and assuming 8000 leukocytes per microliter of blood. If no gametocytes were detected in 100 fields, additional fields were examined until a gametocyte was seen or a maximum observation time of 60 min was reached.

\section{Preparation of thick blood films}

Thick blood films were prepared from $10 \mu \mathrm{L}$ of blood from each dilution. The films were stained with 5\% Giemsa stain without prior methanol fixation for $10 \mathrm{~min}$ and carefully rinsed with water. The films were evaluated using the same protocol as that used for the MF preparations. 


\section{Reverse transcriptase polymerase chain reaction}

RT-PCR was conducted based on the methods of Mlambo et al [12], Babiker et al [13], and Menegon et al [14] for the gametocyte specific genes $P f s 25$ and $P f g 377$ using the same primer sequences as described in these publications. Total RNA was extracted from $1.5 \mathrm{~mL}$ blood from each dilution using the Qiagen Blood Kit following manufacturers instructions. RNA was eluted in $60 \mu \mathrm{L}$ of RNAse free water. RNA was subjected to DNAse treatment (DNA-free Kit, Ambion Applied Biosystems). Total RNA was eluted with $60 \mu \mathrm{L}$ of RNAse free water and DNAse treated using the Ambion DNA-free Kit (Ambion Applied Biosciences). The total RNA was quantified on a spectrophotometer (Nanodrop 1000, Thermo Fisher Scientific Inc.). RNA was subjected to reverse transcription directly without intermediate storage. cDNA was obtained from $5.5 \mu \mathrm{L}$ of the total RNA eluate using reagents from a Superscript III first strand kit (Invitrogen) following manufacturers instructions with sequence specific primers $P f s 25-\mathrm{R}$ (5'-AATTCTTACATTATAAAAAAGCATACTC-3') for the Pfs25 gene and Pfg377-R3D1 (5'-GATGAAAGGGATATATCACCTCACAAT GTG-3') and Pfg377-R3R2 (5'-GTCATGATTTTCTTCTCCTTCGGATATGG-3') for the $P f g 377$ gene respectively.

PCR was conducted immediately following reverse transcription using reagents from the Platinum Taq Polymerase Kit (Invitrogen). The first PCR was conducted using 2 $\mu \mathrm{L}$ of cDNA template in $18 \mu \mathrm{L}$ of master mix containing primers $P f s 25-\mathrm{R}$ and $P f$ s25-F (5'-ATCGATATGAATA AACT TTACAGTTTGTTTCT-3') for Pfs25 and Pfg377-R3D1 and Pfg377-R3R2 for Pfg377 respectively. The nested PCR was conducted using $2 \mu \mathrm{L}$ of the product from the first PCR. For Pfs 25 primers $P f s 25-1$ (5'-TAATGCGAAAGTTACCG TGG-3') and Pfs25-2 (5'-TCCATCAACAGCTTTACAGG-3') were used. For $P f g 377$ primers $P f g 377-R 3 D 2$ (5'-CCATAGGAATATTACACCATATCATGT G-3') and Pfg377-R3R1 (5'TATGGTGATAAATGAGGAGTGTCCCC TTAC-3') were used. The first PCR was conducted on two separate PTC 100 Thermocyclers (MJ Research Inc.) using the cycling conditions for each gene as described previously by Babicker and Menegon with 35 cycles each [13,14]. No gels were run for the product from the first PCR and products were directly subjected to nested PCR. The nested PCR was conducted on a Rotorgene RG3000 thermocycler (Corbett Research) using the following cycling conditions for Pfs25: Hold: $95^{\circ} \mathrm{C}, 5 \mathrm{~min}$; Cycling: $95^{\circ} \mathrm{C}, 15 \mathrm{~s} ; 50{ }^{\circ} \mathrm{C}$, 20s; $72{ }^{\circ} \mathrm{C}$, 40 s; for 40 cycles; Hold: $72{ }^{\circ} \mathrm{C}$, 30s; Melt: $72{ }^{\circ} \mathrm{C}$ to $99^{\circ} \mathrm{C}$; Hold: $40^{\circ} \mathrm{C}$, 30 s and for $P f g 377$ : Hold: $94^{\circ} \mathrm{C}, 5$ min; Cycling: $94^{\circ} \mathrm{C} 35 \mathrm{~s}, 55^{\circ} \mathrm{C}, 30 \mathrm{~s} ; 72^{\circ} \mathrm{C}, 60$ s for 40 cycles; Hold $72^{\circ} \mathrm{C}$, 30 s Melt: $72^{\circ} \mathrm{C}$ to $99^{\circ} \mathrm{C}$; Hold: $40^{\circ} \mathrm{C}$, 30s. PCR products were verified by melt curve analysis and gel electrophoresis in $1.5 \%$ agarose gel, containing ethidium bromide at $100 \mathrm{~V}$ for $60 \mathrm{~min}$. The gels were visualized in a UV transluminator (Versadoc, Model 3000, Biorad).

\section{Data analysis}

Only dilutions with a calculated gametocyte density of < $100 \mu \mathrm{L}^{-1}$ were used for comparison of the three techniques so that each of the six dilution series had one dilution in each of the following ranges: $10^{1}-10^{2}, 10^{0}-10^{1}, 10^{-1}-10^{0}$, and $10^{-2}-10^{-1} \mu \mathrm{L}^{-1}$. The limit of detection for each method in each dilution series was defined as the lowest gametocyte density where detection was positive. Detection limits were compared by using a nonparametric significance test (Wilcoxon's matched pairs test). The sensitivity of each technique for a given specified range of gametocyte density was defined as the fraction of gametocyte tests carried out in that range that were positive. In the case of RT-PCR a positive test was defined as the positive detection of at least one of the two genes being assayed.

\section{Results}

Gametocytes were observed on the MF and TBF preparations as shown in Figure 1.

The increase in observed mean gametocyte number per field on the MF preparations was 12 (range 5-26) fold as
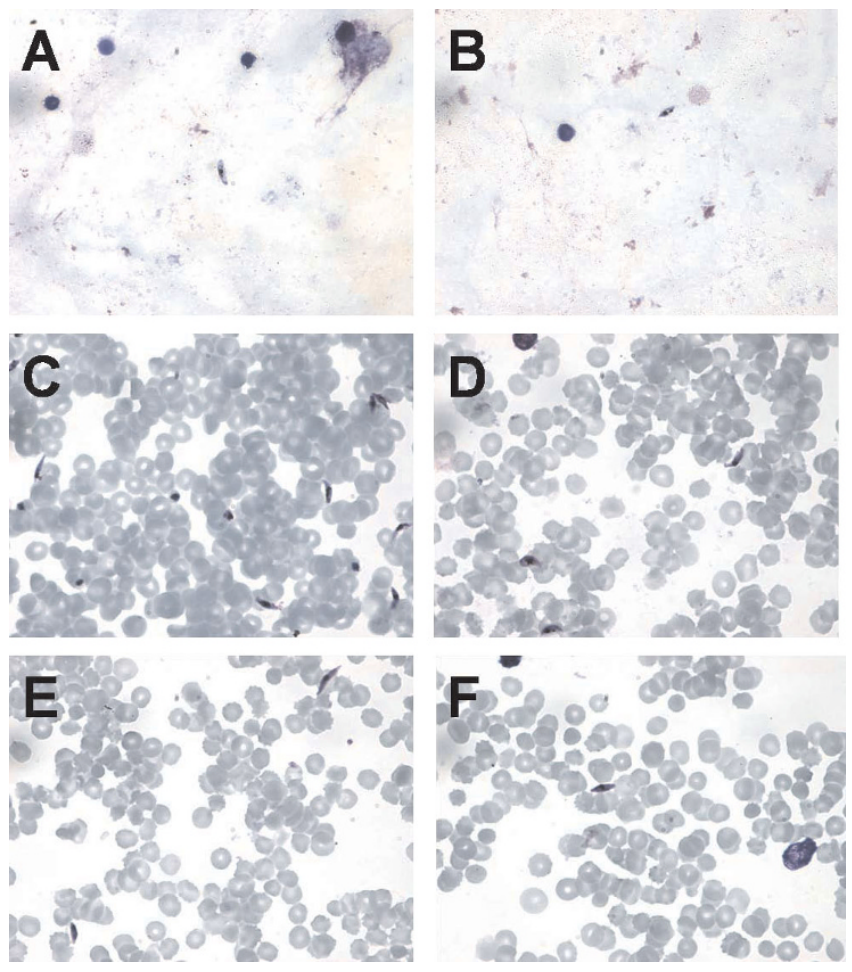

Figure I

Gametocytes on thick blood film (TBF) and magnetically fractionated (MF) preparations. Gametocytes as observed on TBF in the ranges of $10^{1}-10^{2}(A)$ and $10^{0}-10^{1}$ (B) as compared with gametocytes as observed on MF preparations in the ranges of $10^{1}-10^{2}(\mathrm{C}), 10^{0}-10^{1}(\mathrm{D}), 10^{-1}-10^{\circ}(\mathrm{E})$ and $10^{-2}-10^{-1}(\mathrm{~F})$. Images were obtained on a Nikon Eclipse TE2000 -N Microscope with a $1000 \times$ optical magnification with a Nikon LH-MI00CB-I Camera. 
compared to the corresponding TBF preparations, while the increase in observed mean gametocyte density was 319 (range 71-1387) fold (Figure 2).

The mean number of fields observed in 60 minutes for the gametocyte negative slides was $1740(+/-228)$ corresponding to a mean blood volume of 2.8 (SD 0.8) $\mu \mathrm{L}$ if based on 8000 leukocytes $\mu \mathrm{L}^{-1}$.

The mean total RNA from all 24 extractions was 79.9 (SD 20.1) ng/ $\mu \mathrm{L}$ in $60 \mu \mathrm{L}$ of RNAse free water. Parasite RNA did not make a difference to the mean RNA content, since leukocyte RNA formed the vast majority of the RNA present.

RT-PCR showed bands of similar sized product, to that described by Babiker et al [13] and Mlambo et al [12], and Menegon et al [14] in all experiments down to the range of $10^{-1}-10^{0}$ gametocytes $\mu \mathrm{L}^{-1}$ (Figure 3 ). The limits of detection for three methods for each dilution series are shown in Figure 4.

In pairwise comparison, the limits of detection for the MF technique were significantly lower than those for the TBF technique ( $\mathrm{p}=0.031)$ with the same trend when comparing RT-PCR to TBF $(\mathrm{p}=0.062)$. There was no significant difference between the detection limits for MF and RTPCR.

Table 1 shows the sensitivities for each method for each of the gametocyte density ranges. Both MF and RT-PCR showed $100 \%$ sensitivity for the detection of gametocytes down to concentrations in the range $10^{-1}-10^{0} \mu \mathrm{L}^{-1}$. At lower concentrations, the sensitivity of both techniques decreased. By contrast the sensitivity of the TBF technique was below $100 \%$ in the concentration range $10^{0}-10^{1}$ exhibiting $0 \%$ sensitivity in the $10^{-2}-10^{-1}$ range. The mean observation time was considerably less for the MF technique than for the TBF technique (see Table 1).

Detection limits of the 3 different methods are compared in Figure 4. Sensitivities and slide observation times required to detect the first gametocyte are compared in Table 1.

\section{Discussion}

In the present study, MF showed an improved gametocyte limit of detection as compared with TBF and a similar limit to that of RT-PCR. Identification of gametocytes was easier in the thin film-like MF preparations compared with the TBF preparations. Unlike current RT-PCR assays, MF assays can easily be adapted to field use and so have potential application to clinical and epidemiological studies in which gametocyte density is a variable of interest.

Several studies have focused on determining the limit of detection for asexual parasite forms and gametocytes on TBF [15-20]. An initial scan of the TBF is often conducted to detect the presence of malaria parasites before determining parasite density. The number of fields examined in the initial scan typically varies between 30 and 500 [1517]. The volume of blood observed in a TBF is dependent on the preparation method and the technique of the
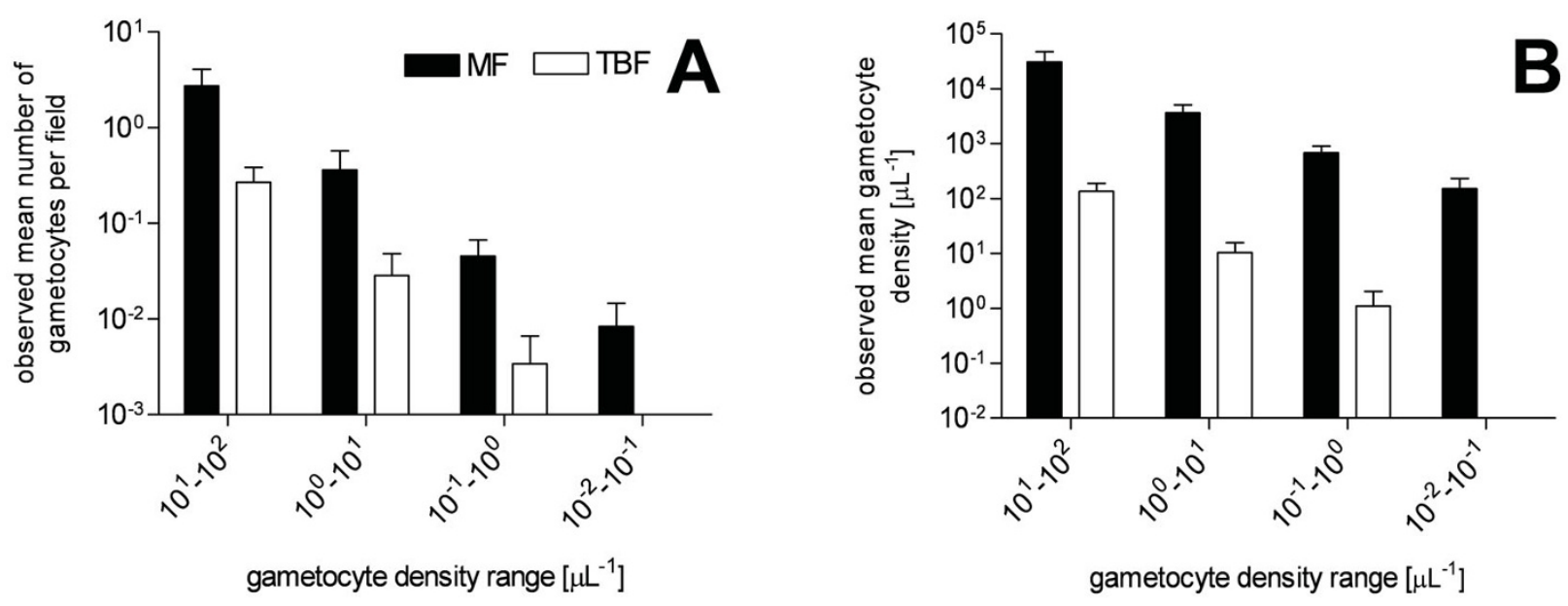

Figure 2

Observed gametocyte increase on MF preparations as compared to TBF preparations. Panel A: Mean gametocyte number per high power microscope field for each of the gametocyte density ranges for MF and TBF preparations. Panel B: Mean gametocyte density observed with MF and TBF for each of the gametocyte density ranges. 


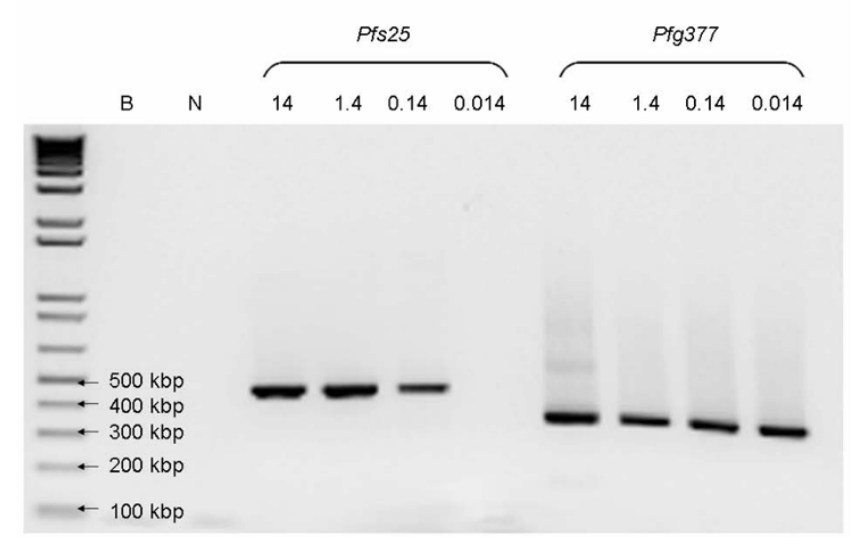

Figure 3

RT-PCR for Pfs25 and Pfg377. Example gel showing bands for $P f s 25$ and $P f g 377$ from one of the dilution series used in this study. $\mathrm{B}=$ Blank, $\mathrm{N}=$ uninfected blood. The numbers above the other lanes are the gametocyte densities $\left(\mu L^{-1}\right)$ in the dilutions used in this experiment.

microscopist. The TBF contains less blood per field on the edges while the largest blood volume per field is found in the centre. Different studies report that scanning 100 fields on a TBF corresponds to between 0.28 and $1 \mu \mathrm{L}$ of blood [18-20]. In the present study 100 fields on a TBF preparation corresponded to a mean of $0.16 \mu \mathrm{L}$ blood. However, if necessary, blood volumes up to $2.8 \mu \mathrm{L}$ were assessed.

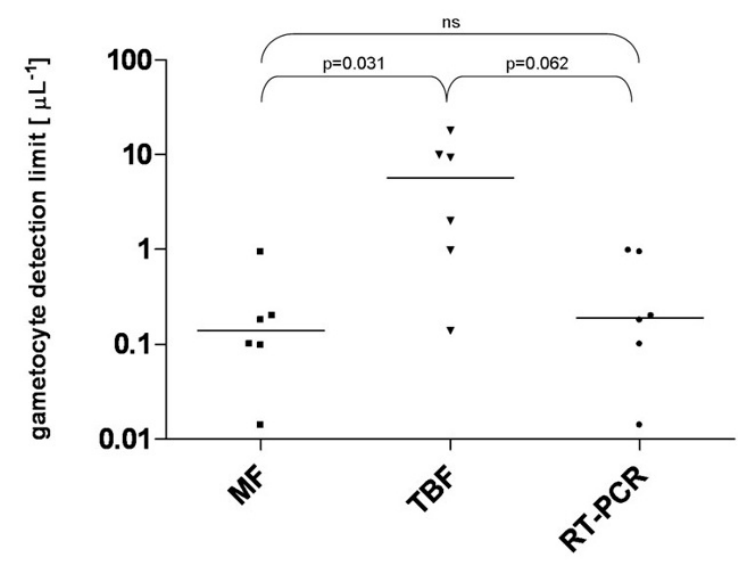

Figure 4

Detection limits for gametocytes using magnetic fractionation (MF), thick blood film (TBF), and RT-

PCR techniques. Wilcoxon's matched pairs test was used as the significance test.
Using Poisson statistics, it can be shown that there is an approximate $95 \%$ probability that a gametocyte will be present in an observed volume $(\mathrm{V})$ of blood if the gametocyte density in the source blood is equal to $3 / \mathrm{V}$. Therefore for a reliable detection of a parasite density of $1 \mu \mathrm{L}^{-1}$, a volume of at least $3 \mu \mathrm{L}$ has to be examined on the TBF.

Such volumes are rarely achieved in routine microscopy for malaria diagnosis. Previous studies have reported that actual detection limits are higher than the calculated theoretical limit based on Poisson statistics [19-22]. A realistic lower detection limit of $50 \mu \mathrm{L}^{-1}$ when examining a blood volume of $1 \mu \mathrm{L}$ on a TBF is in accord with our findings and the literature [23].

Estimates for the limit of detection for parasites on TBF range from $10^{0}-10^{2} \mu \mathrm{L}^{-1}[20,24-27]$, but sensitivity is reported to drop to near zero for densities below $10 \mu \mathrm{L}^{-1}$ [24]. One study reported the loss of $60-80 \%$ of parasites during thick film preparation [21], but this is likely to be dependent on the particular method of slide preparation [1] and was not observed in the present study. In the specific case of $P$. falciparum gametocytes, the equivalent percentage missing or obscured on TBF has been reported to be $86 \%$ [20]. A recent study claims that TBFs underestimate parasite density by about a factor of 10 [18].

Reported limits of detection for RT-PCR vary widely from $2 \mu \mathrm{L}^{-1}$ [12] to $0.0052 \mu \mathrm{L}^{-1}$ [13]. The latter figure was derived from serial dilutions and a sample volume of 50 $\mu \mathrm{L}$ and $40 \%$ haematocrit. However, based on Poisson statistics there is only a $19 \%$ probability that a gametocyte will be present in a sample of this volume and gametocyte density.

\section{Conclusion}

Magnetic fractionation offers advantages over other techniques of gametocyte detection with a limit of detection below that corresponding to the lowest epidemiologically relevant gametocyte densities. The MF method results in preparations enabling the detection of gametocytes at concentrations two orders of magnitude below that achievable with TBF methods. Although it takes almost an hour to conduct MF as described in the present study, the time required to identify gametocytes in the resultant preparations is much shorter than that required to examine a TBF. RT-PCR samples can be processed in batches but the method cannot compete in time efficiency with either TBF or MF techniques. Owing to the cost of the single-use columns, the price for an MF preparation is about 10 US dollars and is considerably higher than that for a TBF preparation but lower than that for an RT-PCR prep- 
Table I: Observed sensitivities and mean observation times ( $t$ ) for the magnetic fractionation (MF), thick blood film (TBF), and RTPCR methods for the six dilution series in the four ranges of gametocyte density.

\begin{tabular}{|c|c|c|c|c|c|c|}
\hline \multirow[t]{2}{*}{ Gametocyte density range $\left[\mu \mathrm{L}^{-1}\right]$} & \multirow[t]{2}{*}{ Mean gametocyte density (range) $\left[\mu \mathrm{L}^{-1}\right]$} & \multicolumn{2}{|c|}{ MF } & \multicolumn{2}{|c|}{ TBF } & \multirow{2}{*}{$\begin{array}{c}\text { PCR } \\
\begin{array}{c}\text { Sensitivity } \\
{[\%]}\end{array}\end{array}$} \\
\hline & & $\begin{array}{c}\text { Sensitivity } \\
{[\%]}\end{array}$ & $\mathrm{t}[\mathrm{min}]$ & $\begin{array}{c}\text { Sensitivity } \\
{[\%]}\end{array}$ & $\mathrm{t}[\mathrm{min}]$ & \\
\hline $10^{1}-10^{2}$ & $57(14-97)$ & 100 & $<1$ & 100 & 2 & 100 \\
\hline $100-101$ & $5.7(1.4-9.7)$ & 100 & $<1$ & 83 & 17 & 100 \\
\hline $10^{-1}-10^{0}$ & $0.57(0.14-0.97)$ & 100 & 3 & 33 & 46 & 100 \\
\hline $10^{-2}-10^{-1}$ & $0.057(0.014-0.097)$ & 50 & 33 & 0 & 60 & 33 \\
\hline
\end{tabular}

aration. With magnetic deposition microscopy, the cost for a magnetically fractionated sample can be reduced to the cost of a standard thick film.

\section{Abbreviations}

MACS: Magnetically Activated Cell Sorting; MDM: Magnetic Deposition Microscopy; MF: Magnetic fractionation; RT-PCR: Reverse Transcriptase Polymerase Chain Reaction; TBF: Thick blood film.

\section{Competing interests}

The authors declare that they have no competing interests.

\section{Authors' contributions}

SK did the experimental work including cell culture, magnetic fractionations, RT-PCR and slide analysis. SK, TD and TSP conceived of the study. SK, TD, TSP prepared the manuscript.

\section{Acknowledgements}

The authors acknowledge Peter Zimmerman and Maciej Zborowski for critical review of the contents of the manuscript. TMED is supported by a National Health and Medical Research Council of Australia Practioner Fellowship.

\section{References}

I. Trape JF: Rapid evaluation of malaria parasite density and standardization of thick smear examination for epidemiological investigations. Trans $R$ Soc Trop Med Hyg 1985, 79(2): $181-184$.

2. Carter RGR: Infectiousness and gamete immunization in malaria. In: Research in Malaria. Volume 3. Edited by: J K. AcademicPress; 1 980:263-298.

3. Schneider P, Bousema JT, Gouagna LC, Otieno S, Vegte-Bolmer M van de, Omar SA, Sauerwein RW: Submicroscopic Plasmodium falciparum gametocyte densities frequently result in mosquito infection. Am J Trop Med Hyg 2007, 76(3):470-474.

4. Hackett S, Hamzah J, Davis TM, St Pierre TG: Magnetic susceptibility of iron in malaria-infected red blood cells. Biochim Biophys Acta 2009, 1792(2):93-99.

5. Nalbandian RM, Sammons DW, Manley M, Xie L, Sterling CR, Egen NB, Gingras BA: A molecular-based magnet test for malaria. Am J Clin Pathol 1995, 103(I):57-64.

6. Paul F, Roath S, Melville D, Warhurst DC, Osisanya JO: Separation of malaria-infected erythrocytes from whole blood: use of a selective high-gradient magnetic separation technique. Lancet 198I, 2(8237):70-7I.

7. Ahn SY, Shin MY, Kim YA, Yoo JA, Kwak DH, Jung YJ, Jun G, Ryu SH, Yeom JS, Ahn JY, et al: Magnetic separation: a highly effective method for synchronization of cultured erythrocytic Plasmodium falciparum. Parasitol Res 2008, 102(6): | 195-1200.
8. Ribaut C, Berry A, Chevalley S, Reybier K, Morlais I, Parzy D, Nepveu F, Benoit-Vical F, Valentin A: Concentration and purification by magnetic separation of the erythrocytic stages of all human Plasmodium species. Malar J 2008, 7:45.

9. Staalsoe T, Giha HA, Dodoo D, Theander TG, Hviid L: Detection of antibodies to variant antigens on Plasmodium falciparuminfected erythrocytes by flow cytometry. Cytometry 1999, 35(4):329-336.

10. Zimmerman PA, Thomson JM, Fujioka H, Collins WE, Zborowski M: Diagnosis of malaria by magnetic deposition microscopy. Am J Trop Med Hyg 2006, 74(4):568-572.

II. Karl S, David M, Moore L, Grimberg BT, Michon P, Mueller I, Zborowski M, Zimmerman PA: Enhanced detection of gametocytes by magnetic deposition microscopy predicts higher potential for Plasmodium falciparum transmission. Malar J 2008, 7:66.

12. Mlambo G, Vasquez $Y$, LeBlanc R, Sullivan D, Kumar N: A filter paper method for the detection of Plasmodium falciparum gametocytes by reverse transcription polymerase chain reaction. Am J Trop Med Hyg 2008, 78(I): I I4-II6.

13. Babiker HA, Abdel-Wahab A, Ahmed S, Suleiman S, Ranford-Cartwright L, Carter R, Walliker D: Detection of low level Plasmodium falciparum gametocytes using reverse transcriptase polymerase chain reaction. Mol Biochem Parasitol 1999, 99(I): $143-148$.

14. Menegon M, Severini C, Sannella A, Paglia MG, Sangare D, AbdelWahab A, Abdel-Muhsin AA, Babiker H, Walliker D, Alano P: Genotyping of Plasmodium falciparum gametocytes by reverse transcriptase polymerase chain reaction. Mol Biochem Parasitol 2000, IIII(I):|153-16I.

15. Humar A, Harrington MA, Kain KC: Evaluation of a non-isotopic polymerase chain reaction-based assay to detect and predict treatment failure of Plasmodium vivax malaria in travellers. Trans R Soc Trop Med Hyg 1997, 9 I (4):406-409.

16. Seesod N, Nopparat P, Hedrum A, Holder A, Thaithong S, Uhlen M, Lundeberg J: An integrated system using immunomagnetic separation, polymerase chain reaction, and colorimetric detection for diagnosis of Plasmodium falciparum. Am J Trop Med Hyg 1997, 56(3):322-328.

17. Wongsrichanalai C, Chuanak N, Tulyayon S, Thanoosingha N, Laoboonchai A, Thimasarn K, Brewer TG, Heppner DG: Comparison of a rapid field immunochromatographic test to expert microscopy for the detection of Plasmodium falciparum asexual parasitemia in Thailand. Acta Trop 1999, 73(3):263-273.

18. Bejon P, Andrews L, Hunt-Cooke A, Sanderson F, Gilbert SC, Hill AV: Thick blood film examination for Plasmodium falciparum malaria has reduced sensitivity and underestimates parasite density. Malar J 2006, 5: 104.

19. Greenwood BM, Armstrong JR: Comparison of two simple methods for determining malaria parasite density. Trans $R$ Soc Trop Med Hyg 199I, 85(2): I86- 88.

20. Warhurst DC, Williams JE: ACP Broadsheet no 148. July 1996. Laboratory diagnosis of malaria. I Clin Pathol 1996, 49(7):533-538.

21. Dowling MA, Shute GT: A comparative study of thick and thin blood films in the diagnosis of scanty malaria parasitaemia. Bull World Health Organ 1966, 34(2):249-267.

22. Wongsrichanalai C, Barcus MJ, Muth S, Sutamihardja A, Wernsdorfer $\mathrm{WH}$ : A review of malaria diagnostic tools: microscopy and 
rapid diagnostic test (RDT). Am J Trop Med Hyg 2007, 77(6 Suppl):II9-127.

23. Moody A: Rapid diagnostic tests for malaria parasites. Clin Microbiol Rev 2002, I 5(I):66-78.

24. Craig MH, Sharp BL: Comparative evaluation of four techniques for the diagnosis of Plasmodium falciparum infections. Trans R Soc Trop Med Hyg 1997, 9 I (3):279-282.

25. Hanscheid T: Diagnosis of malaria: a review of alternatives to conventional microscopy. Clin Lab Haematol 1999, 2I(4):235-245.

26. Milne LM, Kyi MS, Chiodini PL, Warhurst DC: Accuracy of routine laboratory diagnosis of malaria in the United Kingdom. J Clin Pathol 1994, 47(8):740-742.

27. Payne D: Use and limitations of light microscopy for diagnosing malaria at the primary health care level. Bull World Health Organ 1988, 66(5):621-626.

Publish with Bio Med Central and every scientist can read your work free of charge

"BioMed Central will be the most significant development for disseminating the results of biomedical research in our lifetime. "

Sir Paul Nurse, Cancer Research UK

Your research papers will be:

- available free of charge to the entire biomedical community

- peer reviewed and published immediately upon acceptance

- cited in PubMed and archived on PubMed Central

- yours - you keep the copyright

Submit your manuscript here:

http://www.biomedcentral.com/info/publishing_adv.asp 